\title{
An ultra-high speed whole slide image viewing system
}

\author{
Yukako Yagi $^{\mathrm{a}, \mathrm{b}, *}$, Shigeatsu Yoshioka ${ }^{\mathrm{c}}$, Hiroshi Kyusojin ${ }^{\mathrm{c}}$, Maristela Onozato ${ }^{\mathrm{a}, \mathrm{b}}$, Yoichi Mizutani ${ }^{\mathrm{c}}$, \\ Kiyoshi Osato ${ }^{\mathrm{c}}$, Hiroaki Yada ${ }^{\mathrm{c}}$, Eugene J. Mark ${ }^{\mathrm{a}, \mathrm{b}}$, Matthew P. Frosch ${ }^{\mathrm{a}, \mathrm{b}}$ and David N. Louis ${ }^{\mathrm{a}, \mathrm{b}}$ \\ ${ }^{a}$ Pathology Service, Massachusetts General Hospital, Boston, MA, USA \\ ${ }^{\mathrm{b}}$ Department of Pathology, Harvard Medical School, Boston, MA, USA \\ ' Sony Corporation, Tokyo, Japan
}

\begin{abstract}
Background: One of the goals for a Whole Slide Imaging (WSI) system is implementation in the clinical practice of pathology. One of the unresolved problems in accomplishing this goal is the speed of the entire process, i.e., from viewing the slides through making the final diagnosis. Most users are not satisfied with the correct viewing speeds of available systems. We have evaluated a new WSI viewing station and tool that focuses on speed.

Method: A prototype WSI viewer based on PlayStation ${ }^{\circledR} 3$ with wireless controllers was evaluated at the Department of Pathology at MGH for the following reasons: 1. For the simulation of signing-out cases; 2. Enabling discussion at a consensus conference; and 3. Use at slide seminars during a Continuing Medical Education course.

Results: Pathologists were being able to use the system comfortably after 0-15 min training. There were no complaints regarding speed. Most pathologists were satisfied with the functionality, usability and speed of the system. The most difficult situation was simulating diagnostic sign-out.

Conclusions: The preliminary results of adapting the Sony PlayStation ${ }^{\circledR} 3\left(\mathrm{PS}^{\circledR}\right)$ as an ultra-high speed WSI viewing system were promising. The achieved speed is consistent with what would be needed to use WSI in daily practice.
\end{abstract}

Keywords: Ultra-high speed viewing system, whole slide imaging, PS3 ${ }^{\circledR}$

\section{Introduction}

The ability to digitize histopathology slides automatically, rapidly and with high resolution has been pursued by numerous investigators around the world. One of the goals is the implementation of this technology in clinical practice. A pending issue is the time involved for the entire process, from viewing the slides through making the final diagnosis. While scanning time is faster and the performance characteristics of

\footnotetext{
*Corresponding author: Yukako Yagi, PhD, The MGH Pathology Imaging and Communication Technology (PICT) Center, Massachusetts General Hospital, 101 Merrimac St. Suite 820, Boston, MA 02114, USA. Tel.: +1 617643 5162; Fax: +1 617643 7901; E-mail: yyagi@partners.org.
}

available scanners are generally acceptable, viewing speed is still not satisfactory for most users. In this paper, we introduce a new ultra-high speed WSI viewing system that addresses this issue.

\section{Why viewing speed is an issue?}

Three years ago, when we looked at a $40 \times$ WSI at standard pathologists' workstations, the image often froze or the software crashed. Today, improvements in network performance and PC specification enable the ability to view $40 \times$ WSI without software crashes. However, the speed of available systems is still not acceptable for routine sign-out. The difficulty to manage large image files, the need for a highly interactive 
display for these images, and fast, reliable image capture remain substantial technical challenges.

\subsection{File size}

A typical, stained microscope slide contains a large amount of potential data. The typical surgical pathologist uses a microscope with a variety of objectives, the most powerful of which is usually a high corrected, $40 \times$ lens with a numerical aperture of approximately 0.9 . While the pathologist never scans the entire slide at $40 \times$, few pathologists are willing to give up the ability to examine tissue at $40 \times$ because it is necessary, in a variety of common diagnostic situations, to examine small areas of the slide at that magnification. The average file size of a $40 \times(0.23 \mathrm{um} /$ pixel $)$ WSI is $1-2$ $\mathrm{GB} / \mathrm{image}$ and image size is $50 \mathrm{~GB}$. For a whole case, total file size may be 10-30 GB because many cases contain 10-20 slides. This is in contrast to the average image size of radiological images, such as $\mathrm{CT}$, which is only $100-200 \mathrm{MB}$.

\subsection{Computer performance}

In general, the PCs used for daily practice are not high-end computers. For example, the computers in the Pathology department at Massachusetts General Hospital (MGH) are currently configured with a Windows XP Professional operating system with an Intel ${ }^{\circledR} \mathrm{Core}^{\mathrm{TM}} 2$ Duo CPU and $4 \mathrm{~GB}$ of RAM. This configuration is not powerful enough to view continuously a WSI scanned at $40 \times$ objective, especially for diagnosis. The reason for this is not only PC performance, but the combination of network and computer performance. Moreover, even with the recommended computer specifications for WSI, it would not be practical to upgrade all PCs in the department. In addition, the institution has guidelines for installing software as well as for upgrading hardware, which would further complicate such a potential solution.

\subsection{Network}

As mentioned above, a high speed and reliable network is required to view a WSI. However, without a dedicated network, it is impossible to maintain the same speed at anytime and anywhere. A dedicated Pathology network within limited areas may be possible in the near future, but not at the present time.
To solve such issues mentioned above, we realized that we needed a viewer with faster viewing speed that could use the current network and at a reasonable cost.

\section{PlayStation ${ }^{\circledR} 3$ technologies}

The PlayStation ${ }^{\circledR} 3\left(\mathrm{PS}^{\circledR}{ }^{\circledR}\right)$ of Sony Corporation, Tokyo, Japan, is a widely known game console that gives users realistic experiences through playing games. Combining advanced motion sensors, a dynamic color changing sphere, vibration feedback, and an easy-to-use button interface, the motion controller delivers a highly immersive gaming experience. We realized that these are the features that were needed for effective WSI viewing. Moreover, the PS ${ }^{\circledR}$ has been designed with capabilities become that of a video game console; its CPU is designed to support a wide range of applications that require real-time processing.

The CPU in the PS ${ }^{\circledR}$ is called a Cell Broadband Engine $^{\mathrm{TM}}$ (Cell BE) which is described in Fig. 1.

Cell Broadband Engine ${ }^{\mathrm{TM}}$ has one control-plane specialized processor (PPU) and eight data-plane specialized processors (SPUs) on the same chip. An important aspect of this design is that the majority of memory requests are handled by instructing the dedicated controller to pre-place data into the local store

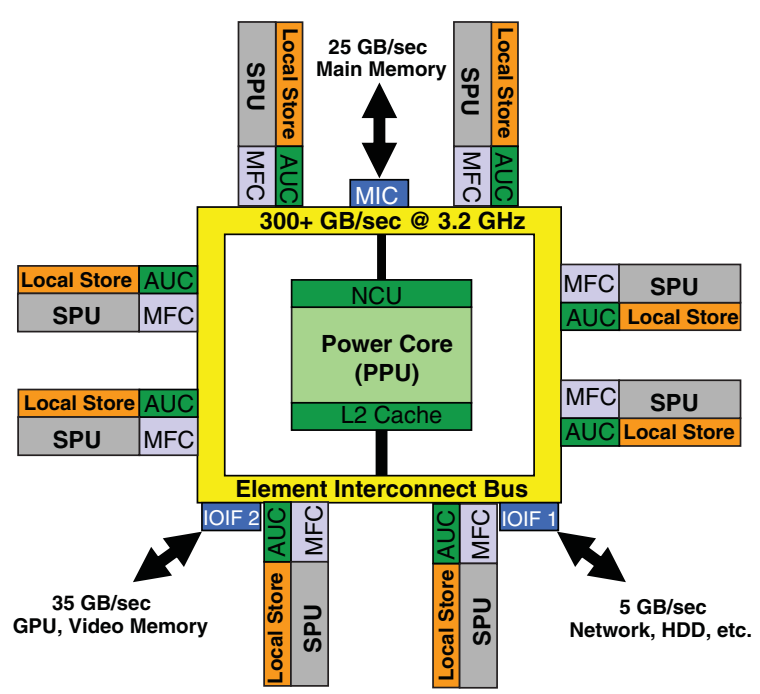

Fig. 1. Cell Broad Band Engine. Cell Broadband Engine has one control-plane specialized processor (PPU), which can be described as a power core and eight $\mathrm{xz}$ data-plane specialized processors (SPUs) on the same chip. 
Table 1

Processor comparison CPU vs. Cell BE vs. GPU*

\begin{tabular}{lccc}
\hline & CPU & Cell BE & GPU \\
& Intel (Core i7) & Sony/IBM/Toshiba & nVidia GTX480 \\
\hline Cores & 4 & 9 & 480 \\
Conditional branching & $\odot$ & $\triangle$ & $\times$ \\
Clock rate & $3.2 \mathrm{GHz}$ & $3.2 \mathrm{GHz}$ & $1.4 \mathrm{GHz}$ \\
Data transfer method & Hardware Cache & DMA & Texturing/rendering \\
\hline
\end{tabular}

of each SPU before it's needed. This is done by DMA so it does not subject the CPU to a heavy overhead. This means that Cell BE can provide nearly full-speed memory access while simultaneously carrying out parallel computation. Graphics processing units (GPUs) have, for many years, powered the display of images and motion on computer displays. GPUs are now powerful enough to do more than just move images across the screen. They are capable of performing high-end computations that are the staple of many engineering activities

The comparison between Cell BE, CPU and GPU are in Table 1. A Cell BE has a mixture of fat and thin cores, which offers the best trade-off between parallelism and controllability. Combined with highly customizable memory-to-memory data transfer using DMA, the Cell BE meets the requirements for a high performance media processing system [1-5]. Besides the main body of PS $3^{\circledR}$, there are many accessories that facilitate playing games more realistically. In this regard, a wireless controller, which is described in Fig. 2, could be one solution for a WSI-human interface. The concept behind featuring shoulder buttons for both the index and middle fingers is to implement two-way directional depth controls with two sets of buttons. This is intended to update controller navigating 3D environments for which the $\mathrm{PS} 33^{\circledR}$ was designed. To compensate for the less stable grip from shifting the middle fingers' placement to the shoulders, grip handles have been added. Simple geometric shapes $\triangle, \bigcirc, x$, and $\square$ rather than letters or number to label it action buttons.

\section{WSI in pathology practice}

To implement WSI in current pathology practice, a WSI system will need to have clear advantages over current practice, which revolves around the utilization of microscopes to examine histopathology slides. The speed of viewing a whole slide image is not the only issue, since one must also quickly select the right slide from many available slides and switch from one slide to another easily. Figure 3 shows a slide tray on which a pathologist often receives slides from the histology laboratory; note that the pathologist can quickly decide which slide to start viewing under a microscope even though the slides may be lined up by slide number. Figure 4 shows how a pathologist uses a microscope, and how a pathologist rapidly switches from one slide to another. We have sometimes used multiple displays
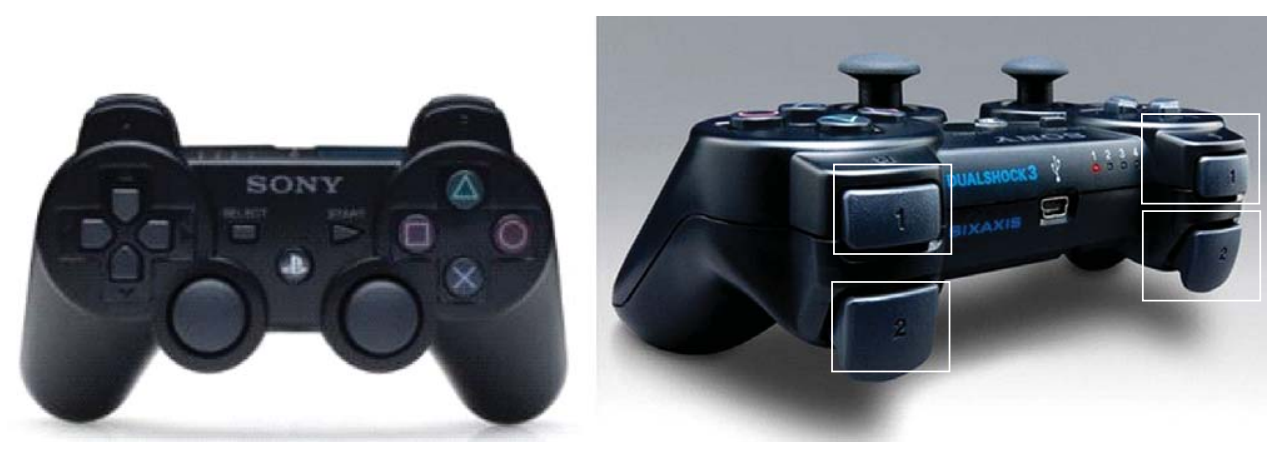

Fig. 2. PS $3{ }^{\circledR}$ Controller. When compared to previous controller, the PS $3{ }^{\circledR}$ Controller added a second pair of shoulder buttons for the middle finger in the basic button configuration. The squares indicate two pairs of shoulder buttons. 


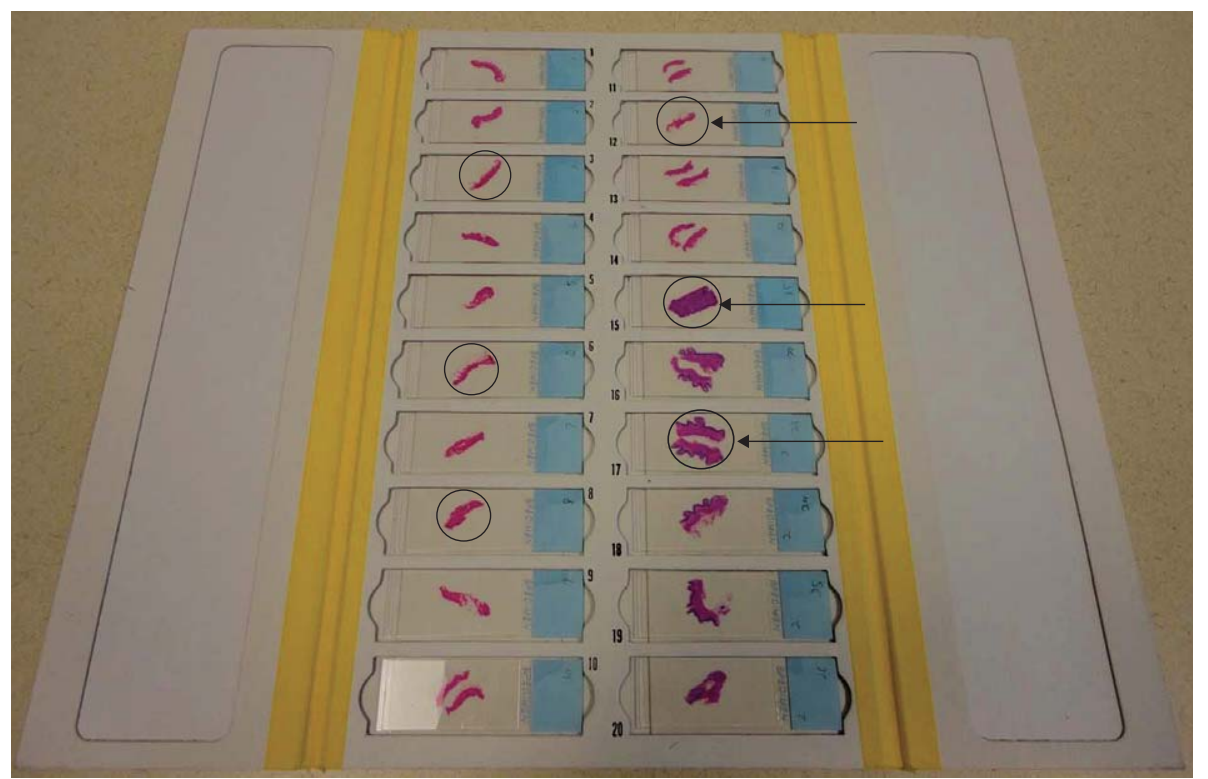

Fig. 3. Slide Tray and Stained Slides. A standard cardboard slide tray that a pathologist receives from the histology laboratory. It is open and contains twenty glass slides with tissue specimens (circles and arrows) that have been stained. In most instances, the slides of a single case number less than twenty and can thus be accommodated in one slide tray.

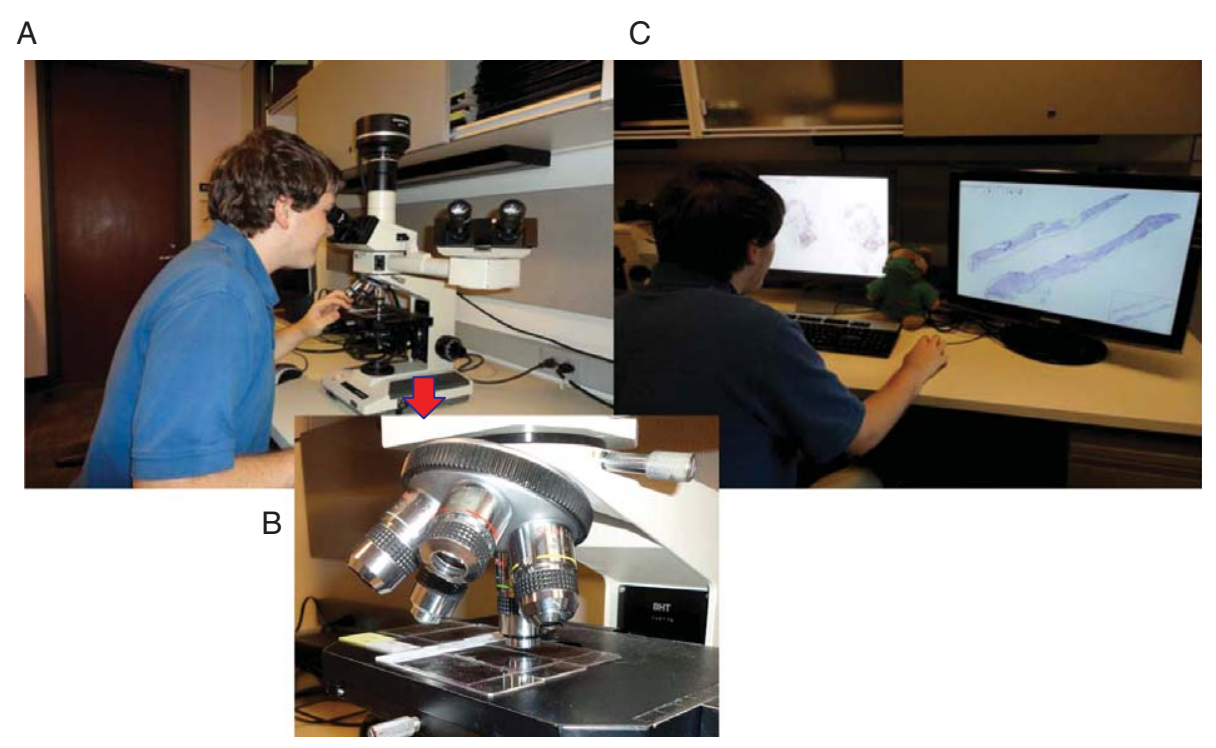

Fig. 4. Microscope usage and Multiple Display. A) A pathologist uses a microscope for diagnosis and uses his/her hand to move the slide for an efficient evaluation. B) Often, multiple slides are placed on the microscope stage to move between slides quickly. C) A WSI viewing station with multiple displays is preferred to view the WSI and make diagnosis with greater speed.

to speed up the viewing of different whole slide images; although this approach provided some improvement in speed, it was not as fast as switching slides on a microscope stage.
The aims of this paper are to determine if the adapted ultra-high speed WSI viewer is acceptable to pathologists; and if acceptable, how effective it is in clinical practice. 


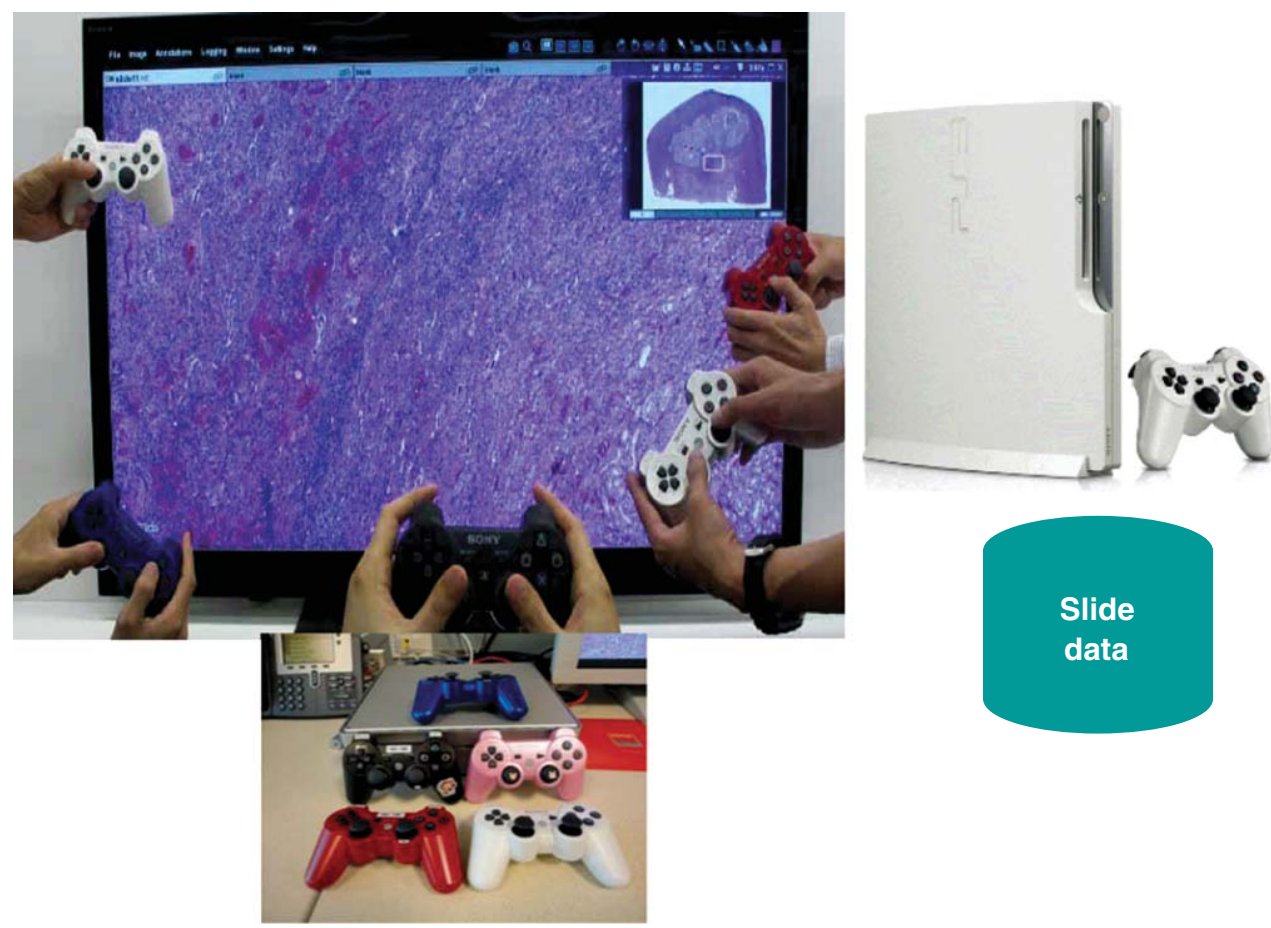

Fig. 5. The prototype WSI viewing system based on PS $3^{\circledR}$; Standalone. Multiple wireless controllers can be used at the same time. The color of the pointer and controller are matched. Personalized controllers are useful. Every participant can use a pointer at anytime. However, only one person at a time can navigate images and control the magnification and location of the region of interest. This role can be easily switched to another participant who knows this because the controller will then vibrate.

\section{Methods and materials}

\subsection{Evaluation criteria}

We evaluated the ultra-high speed WSI viewer system to answer the following five questions:

1. Can it be used for signing out a case?

2. Can it be used at a consensus conference?

3. Can it be used at a slide seminar?

4. How much faster is it than a PC-based viewer working over the public network?

5. Is it user-friendly?

\subsection{System}

A prototype WSI viewing system based on the Sony PS3 ${ }^{\circledR}\left(\mathrm{PS} 3{ }^{\circledR}\right.$-viewer) and wireless controllers was adapted for our study and is depicted in Fig. 5. Multiple wireless controllers can be used at the same time. To evaluate the functionality and operability of
PS3 ${ }^{\circledR}$-viewer, images were stored in a local hard drive. Figure 6 shows the network version. The PS $3^{\circledR}$-viewer accesses the images in the server over the network. We compared the speed with the PC-based WSI viewer system.

\subsection{Materials and methods}

All slides were de-identified by an honest broker system and scanned with an available scanner: either Nanozoomer 2.0-HT (Hamamatsu Corporation, Japan), MiraxScan (3D Histech Ltd., Hungary) or ScanScope CS (Aperio, USA).

For the evaluation of sign-out, 3 breast cases and 3 pancreas cases that had H\&E-stained and immunohistochemical slides were used. For the consensus conference, we used 15 brain tumor slides that were selected from the cases to be reviewed for one week's conference. The slide seminar trials were performed during the 32nd Annual Current Concepts in Surgical Pathology Course organized by the Pathology 


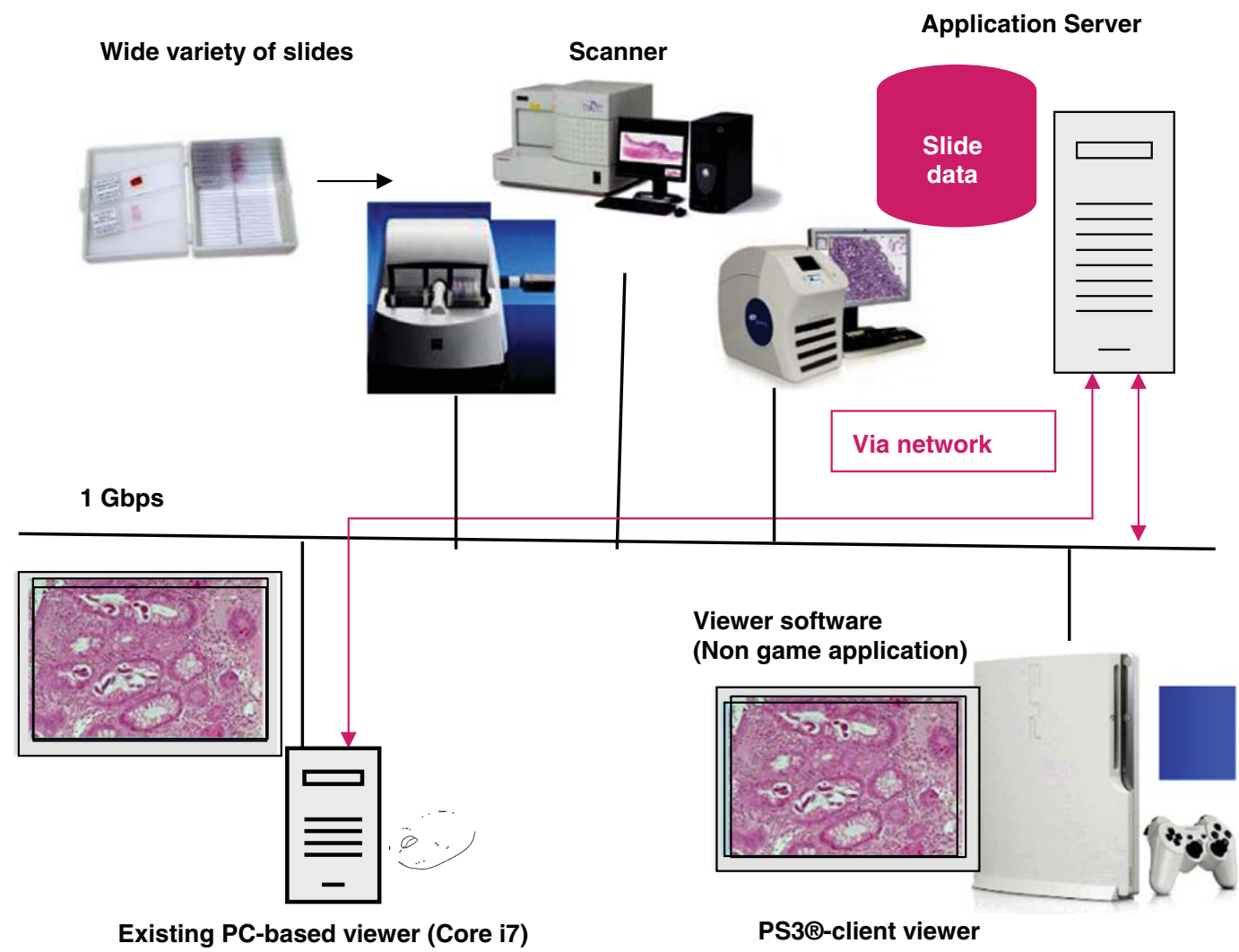

Fig. 6. The prototype WSI viewing system based on PS $3^{\circledR}$ : Server Client. Client viewer accesses the images in the storage server located at the Imaging Laboratory at MGH through the application server. Existing PC-based viewers access the images in the same storage server. All scanners send the data to the storage server at the time of scanning.

Department of MGH. All slides had already been scanned to WSI. The file sizes of most images were $0.5 \mathrm{~GB}-2 \mathrm{~GB}$ and the image sizes of the cases were $1 \mathrm{~GB}-50 \mathrm{~GB}$.

\section{Results}

\subsection{Viewer functions}

While pathologists using the system, we found several feature that need to be available. These features, along with their solutions, are listed in Table 2. A graphic user interface to represent a slide tray is shown in Fig. 7. Although the thumbnail size image has enough information to enable pathologists to select a slide to start, it is not enough for the situation wherein a pathologist has to select the most represen- tative slide. By switching the view to that shown in Fig. 8, a pathologist could quickly select which slides to discuss, for instance, at a consensus conference. To show which slides were already reviewed, the thumbnail images of reviewed slides are marked as viewed (arrows).

Pathologists were able to use the system comfortably after $0-15$ minutes of training. Pathologists who played games regularly at home required $0-3$ minutes of training. Pathologists who never played games required a 10-15 minute training session.

There were no complaints regarding the speed and most pathologists were satisfied with the functionality, usability and speed of the system. Multiple wireless controllers worked well at the consensus conference and slide seminars for questions and comments. The most difficult situation was to simulate the signingout of cases because the process to review the slides is case by case, and by individual pathologist; in addition, 
Table 2

Requirements and solutions for whole slide image viewing systems in different activities of surgical pathologists

\begin{tabular}{|c|c|c|}
\hline Activities & Observations and optimal features & Focused points for GUI design \\
\hline Signing out & $\begin{array}{l}\text { - Look at all slides } \\
\text { - Change magnification frequently } \\
\text { - Quick interaction with LIS } \\
\text { - Comparison with IHC/Special Stain }\end{array}$ & $\begin{array}{l}\text { - Quickly moving between slides } \\
\text { - Marking the slides had been reviewed on the slide } \\
\text { tray } \\
\text { - Automatically synchronize multiple images such } \\
\text { as differently stained slides }\end{array}$ \\
\hline Consensus conference & $\begin{array}{l}\text { - Look at selected slides from among many slides } \\
\text { - All/some attendees will use pointers } \\
\text { - Revisit the same areas of interest on a slide } \\
\text { - Image quality to be compared among microscope, } \\
\text { display from video camera attached to microscope, } \\
\text { and WSI }\end{array}$ & $\begin{array}{l}\text { - Quickly selecting a slide from among many slides } \\
\text { using larger thumbnails } \\
\text { - Easy to use pointers for all/some attendees } \\
\text { - Marking areas of interest by all/some attendees so } \\
\text { that the same areas can be revisited } \\
\text { - Image quality ideally to be equivalent or higher } \\
\text { - than microscope } \\
\text { - Ease of switching between operators (organizers) }\end{array}$ \\
\hline Slide seminar & $\begin{array}{l}\text { - Intuitive controls so that no time is needed for } \\
\text { advance training of speakers or audiences } \\
\text { - Every speaker could operate elegantly without } \\
\text { training } \\
\text { - Able to look at the slides in order rather than to } \\
\text { select the slide from a list }\end{array}$ & $\begin{array}{l}\text { - Simple to use } \\
\text { - Quickly move between slides } \\
\text { - Quickly select a slide from among many slides } \\
\text { - Provide support without speaker or audience been } \\
\text { aware that support is even being given }\end{array}$ \\
\hline
\end{tabular}

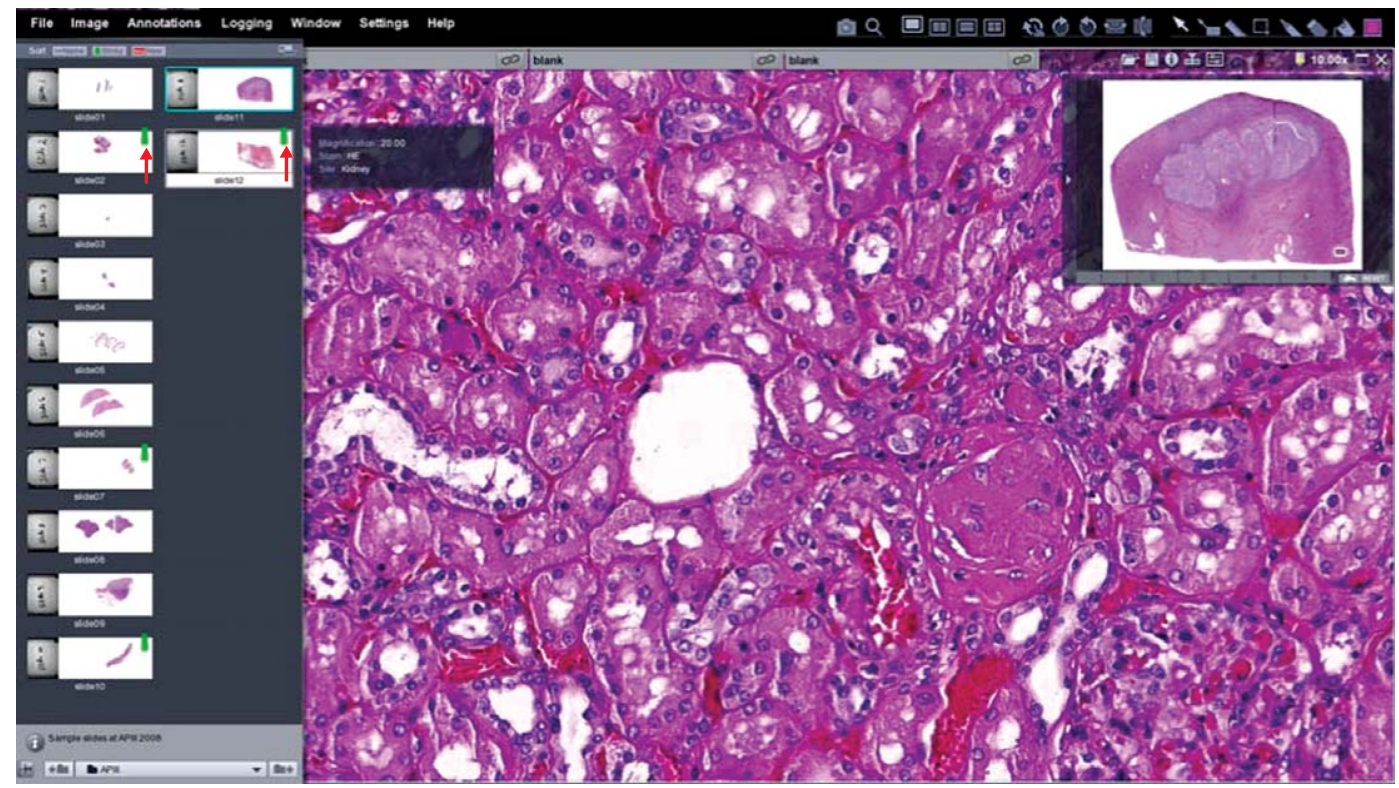

Fig. 7. Slide Selection GUI: Slide Tray. This GUI creates a format comparable to the similar situation to the current sign-out process. The size of the slides on the left side is very close to the size of an actual glass slide when using a 24-26 inch display monitor. Pathologist can check which slides he or she has already observed through an electronic post-it note (arrows). 


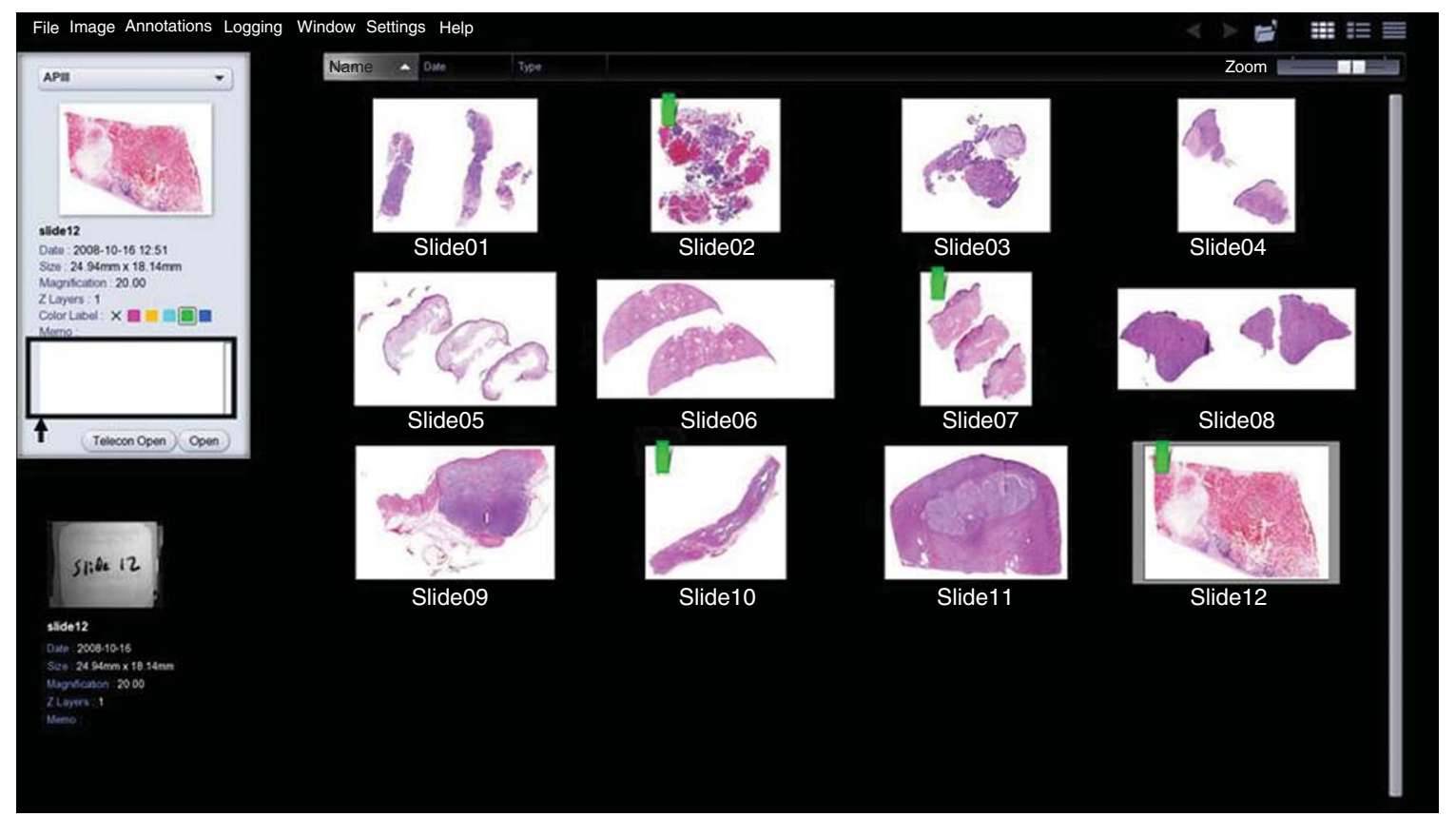

Fig. 8. Slide Selection GUI: This GUI shows more details in the thumbnail images. The GUI also shows posted comments within box, if there are any. And details regarding the slide information can be inserted in the box here shown blank.

the system has to show clearly which slides had been viewed and which slides need to be viewed; and there is the need to potentially see the entire WSI at high magnification.

\subsection{Speed}

Using the $\mathrm{PS} 3{ }^{\circledR}$-viewer, most operations were reflected on the display in about one-sixtieth of a second. The reasons for this speed improvement are a faster processor and an improvement in the prediction of movement and memory management. The tiles to be viewed were prepared beforehand, based on the direction of current movement, and then decoded at ultra high speed. We have compared latency caused by cache miss between three PC based viewers and PS3 ${ }^{\circledR}$ - viewer. The results are shown in Figs. 9 and 10. To measure the latency caused by cache miss, we attached an LED to a computer mouse so that it glowed when clicked. We used this mouse to select a point far from the current view point. Using a high-speed camera, we then measured the lapse between the time the LED lit up and rendering was completed on the

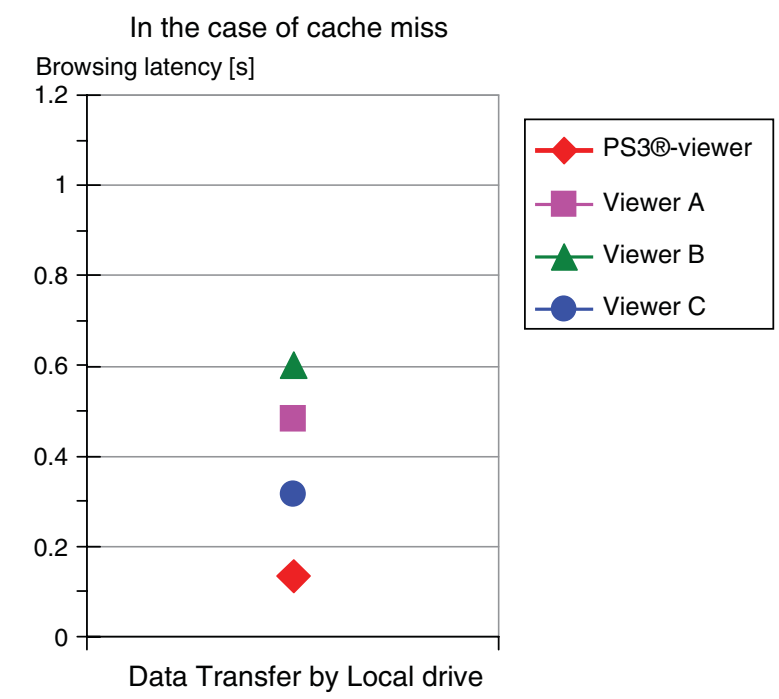

Fig. 9. Access Speed comparison between 3 PC based viewers and PS3 ${ }^{\circledR}$ viewer: Local. Average latency when clicking on the four corners of a thumbnail view.

screen. The PS $3{ }^{\circledR}$-viewer preformed about twice as fast both locally and over the network than other PC-based viewers. 


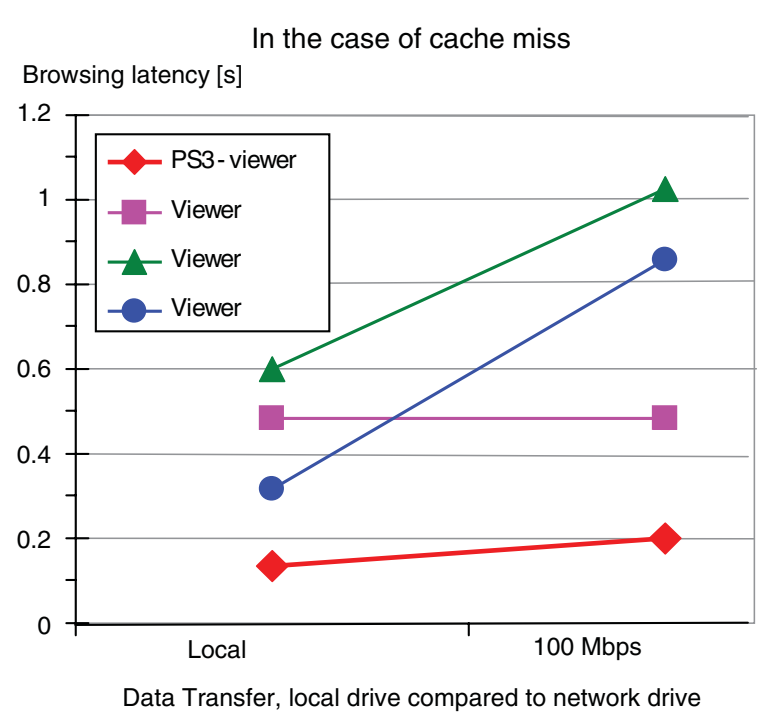

Fig. 10. Access Speed comparison between 3 PC based viewers and PS $3{ }^{\circledR}$ viewer: Network. Over the network, the PS $3{ }^{\circledR}$ viewer showed a favorable result. The difference between local and network is smaller than all other PC based viewers that we have been using.

\section{Discussion}

The speed and user-friendliness of the PS $3^{\circledR}$-viewer was impressive. Another advantage of the system is that it is independent of standard workstations and therefore the IT constraints of the hospital do not apply, including memory size, type of display card, and upgrading of software. Nonetheless, issues of patient confidentiality and encryption requirements would of course need to apply to any system housing patient information.

Thus, our preliminary results using Sony PS $3{ }^{\circledR}$ as an ultra-high speed WSI viewing system were promising, and the speed of imaging viewing and image switching suggested that WSI could be used in daily practice. We nonetheless continue to develop the system to achieve further improvements.

\section{References}

[1] Michael Day and Peter Hofstee, Tutorial: Hardware and Software Architectures for the CELL BROADBAND ENGINE processor, CODES + ISSS Conference, 2005.

[2] Intel Corporation. Intel Core i7-900 Desktop Processor Extreme Edition Series and Intel Core i7-900 Desktop Processor Series Datasheet. 1, 2010.

[3] NVIDIA Corporation. NVIDIA GeForece GTX 480/470/465 GPU Datasheet, 2010.

[4] Sony Corporation, Sony to Showcase, Innovative "Beyond HD" Content Creation Workflows, News Release, 2008.

[5] Eda Hiroki, Cell Decodes 48 MPEG-2 Video Streams at SDTV Resolution, Nikkei Electronics Asia, 2005. 


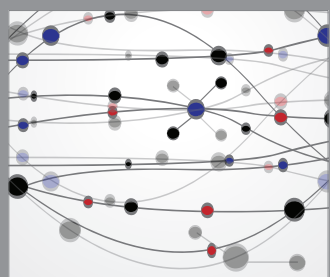

The Scientific World Journal
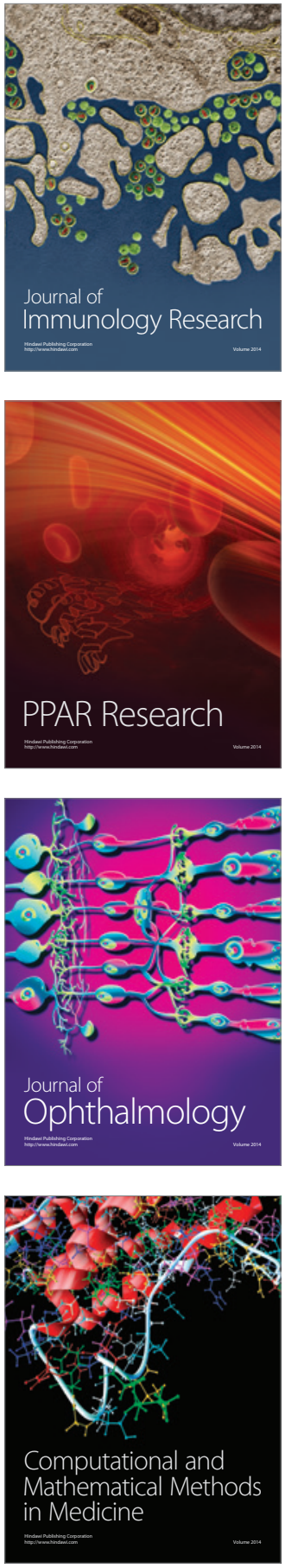

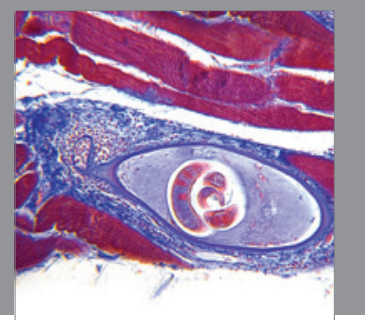

Gastroenterology

Research and Practice
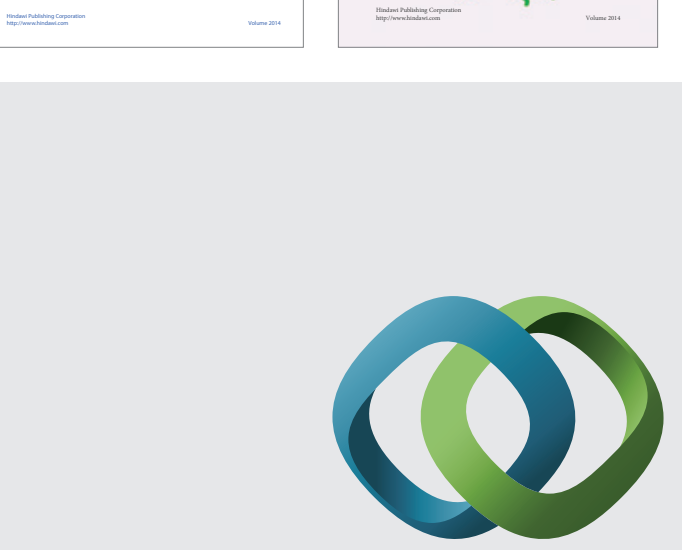

\section{Hindawi}

Submit your manuscripts at

http://www.hindawi.com
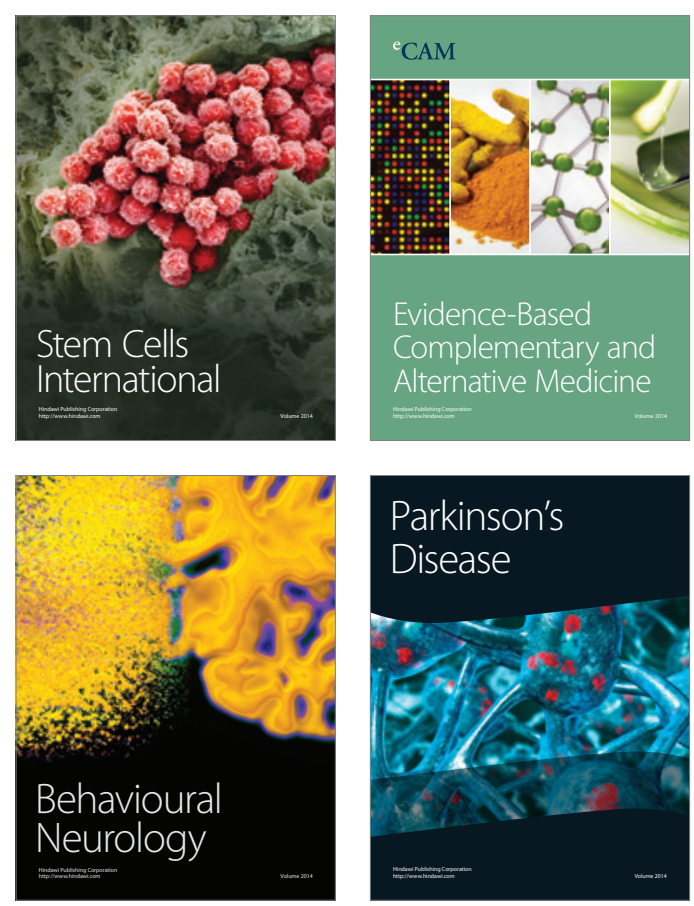

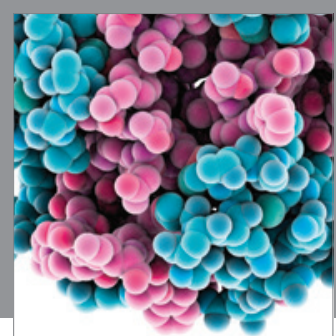

Journal of
Diabetes Research

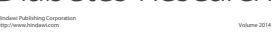

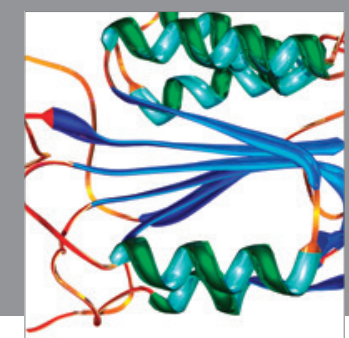

Disease Markers
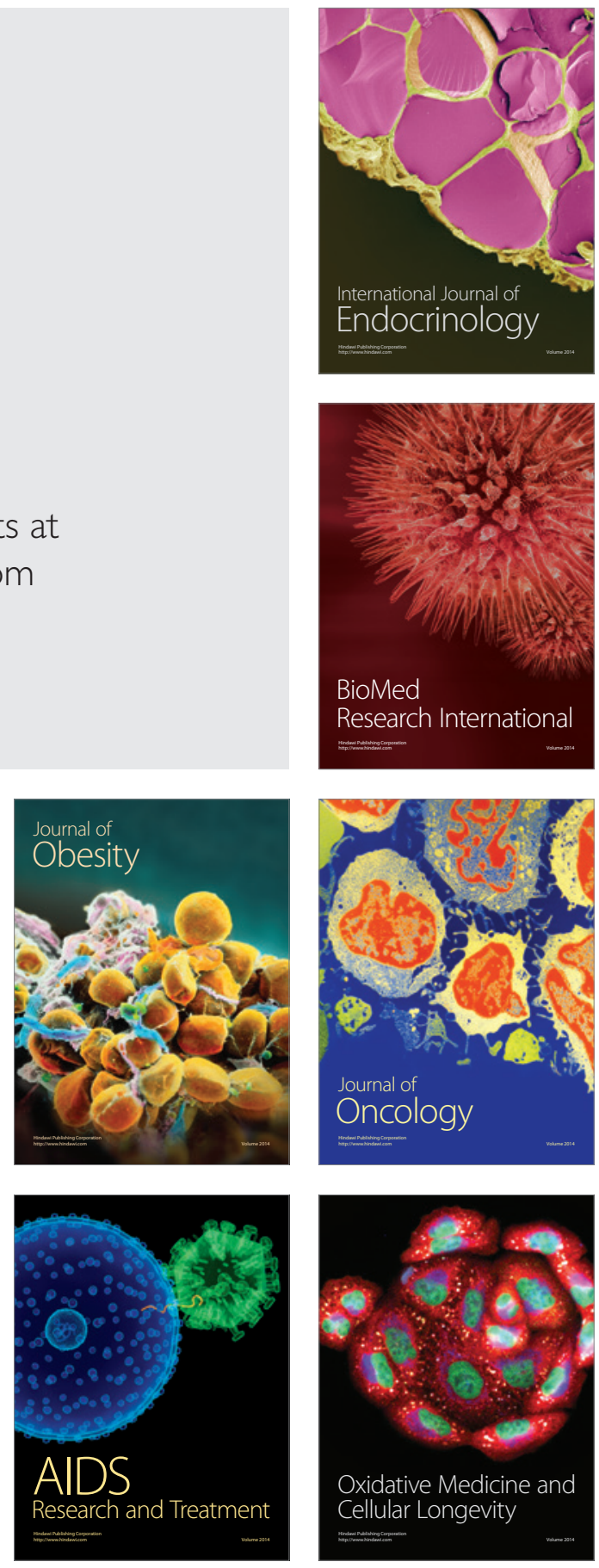